Revue d'histoire de l'Amérique française

Q4. REVUE D'HISTOIRE DE L'AMÉRIQUE FRANÇAISE

\title{
"Les bois engendrent les frimas et les gelées » : comprendre le climat en Nouvelle-France
}

\section{Colin Coates et Dagomar Degroot}

Volume 68, numéro 3-4, hiver-printemps 2015

URI : https://id.erudit.org/iderudit/1033634ar

DOI : https://doi.org/10.7202/1033634ar

Aller au sommaire du numéro

Éditeur(s)

Institut d'histoire de l’Amérique française

ISSN

0035-2357 (imprimé)

1492-1383 (numérique)

Découvrir la revue

Citer cet article

Coates, C. \& Degroot, D. (2015). « Les bois engendrent les frimas et les gelées » : comprendre le climat en Nouvelle-France. Revue d'histoire de l'Amérique française, 68(3-4), 197-219. https://doi.org/10.7202/1033634ar
Résumé de l'article

Cet article juxtapose les reconstructions récentes de l'histoire du climat avec les interprétations de l'époque de la Nouvelle-France. Des auteurs coloniaux ont essayé d'expliquer les hivers beaucoup plus froids que dans la mère patrie. À l'époque des Petits Âges glaciaires, ces auteurs se servent des différences environnementales pour comprendre le climat colonial. Les grandes forêts expliqueraient la longueur et la sévérité hivernales, et les auteurs prédisent que le défrichement des terres allait rapprocher colonie et mère patrie du point de vue du climat. Le réchauffement au cours du XVIII ${ }^{\mathrm{e}}$ siècle a convaincu certains que l'activité humaine avait amélioré le climat. 


\title{
"Les bois engendrent les frimas et les gelées": : comprendre le climat en Nouvelle-France ${ }^{\mathrm{r}}$
}

\author{
Colin COATES \\ Université York \\ Dagomar Degroot \\ Université Georgetown
}

RÉsumÉ - Cet article juxtapose les reconstructions récentes de l'histoire du climat avec les interprétations de l'époque de la Nouvelle-France. Des auteurs coloniaux ont essayé d'expliquer les hivers beaucoup plus froids que dans la mère patrie. À l'époque des Petits Âges glaciaires, ces auteurs se servent des différences environnementales pour comprendre le climat colonial. Les grandes forêts expliqueraient la longueur et la sévérité hivernales, et les auteurs prédisent que le défrichement des terres allait rapprocher colonie et mère patrie du point de vue du climat. Le réchauffement au cours du XVIII siècle a convaincu certains que l'activité humaine avait amélioré le climat.

ABSTRACT - This article juxtaposes current reconstructions of climate history with interpretations from the New France era. During the Little Ice Age, colonial authors attempted to account for winters much colder in the colony than in the mother country, using environmental differences to explain the colonial climate. The authors believed that the expansive forests were probably the source for the length and severity of the winters, and they predicted that large-scale clearings would bring positive climatic changes. The warming trend of the eighteenth century convinced some of them that human activity had in fact contributed to the improvement of the climate.

1. Cette recherche a bénéficié de l'appui du Conseil de recherche en sciences humaines du Canada. Nous tenons à remercier les évaluateurs anonymes pour leurs commentaires fort pertinents. Nous remercions aussi le professeur Fredrik Charpentier Ljungqvist de nous avoir permis de reproduire son graphique, ainsi que nos collègues Teresa Devor, Liza Piper, Graeme Wynn et Madeleine Chartrand pour leurs commentaires et leurs suggestions bibliographiques. Notre collègue Francis Garon, Louise Bienvenue et Andrée Laprise ont eu l'amabilité de bonifier l'expression française de ce texte. 
T a Nouvelle-France, selon les Français, ne devait pas être aussi froide. Située à la même latitude que Paris (presque $49^{\circ} \mathrm{N}$ ), la colonie aurait dû bénéficier en principe d'un climat relativement similaire. D’après le consensus savant de l'époque, la latitude et la hauteur des terres déterminaient le climat. Mais ce n'était certes pas le cas pour la Nouvelle-France, qui était beaucoup plus froide en hiver, et souvent plus chaude en été, que plusieurs régions de la France. Cet aspect de la vie coloniale était perçu de façon très négative; Pierre Boucher y voyait l'une des «incommodités » les plus importantes de la Nouvelle-France, décourageant l'immigration ${ }^{2}$. Ce climat colonial si différent engendrait une question fondamentale: comment comprendre les contrastes entre la Nouvelle-France et la métropole? Pour le jésuite Louis Nicolas, la colonie représentait «un pays étranger, où toutes choses sont différentes du nôtre ${ }^{3}$ ». Ses écrits sur l'histoire naturelle de la colonie indiquent toutefois que les espèces qu'on y trouvait, à quelques exceptions près, n'étaient pas tellement différentes de celles du vieux royaume. Néanmoins, les Français faisaient face au défi de s'établir dans un Nouveau Monde, à la fois si différent et si similaire. Le climat représentait un aspect de première importance qu'ils devaient essayer de comprendre et, au mieux, de modifier.

Au moment du flot migratoire français vers la vallée du Saint-Laurent à la fin du XVII ${ }^{\mathrm{e}}$ siècle, les contemporains ne savaient pas que le climat $\mathrm{y}$ était plus froid qu'au début du siècle, et qu'il se réchaufferait vers le milieu du siècle suivant. Même si le climat devait répondre aux conditions naturelles, certains croyaient que les activités anthropomorphiques pouvaient l'influencer, voire le modifier de façon importante. Par conséquent, le travail de défrichement et de colonisation devait pouvoir améliorer le climat colonial et même faire en sorte que le continent ressemble davantage à l'Europe. Au XVIII ${ }^{e}$ siècle, le changement climatique renforce et justifie la présence française dans le Nouveau Monde. Au même titre que leurs homologues des autres colonies de l'hémisphère, les colons anticipent avec un certain plaisir le réchauffement du climat local ${ }^{4}$. En NouvelleFrance, dès le début du XVII ${ }^{e}$ siècle, les colons s'efforcent de recréer le vieux royaume, et ce travail s'étend jusqu'à la modification du climat local, du moins de leur propre point de vue. La compréhension contemporaine

2. Pierre Boucher, Histoire véritable et naturelle des mours \& productions du pays de la Nouvelle France, vulgairement dite Le Canada (Boucherville, Société historique de Boucherville, 1964 [1664]), 154.

3. François-Marc Gagnon, Nancy Senior et Réal Ouellet, The Codex Canadensis and the Writings of Louis Nicolas (Montréal, Gilcrease Museum et McGill-Queen’s University Press, 2011), 397.

4. Jan Golinski, "American Climate and the Civilization of Nature», dans James Delbourgo et Nicholas Dew, dir., Science and Empire in the Atlantic World (New York, Routledge, 2008), 153-174. 
du climat de la Nouvelle-France reflète le désir de créer une colonie de plus en plus similaire à la mère patrie.

Dans ce texte, nous mettons en relation les données scientifiques concernant les climats anciens avec les perceptions qu'en avaient les contemporains. Nous évoquons, pour débuter, l'historiographie du climat en Nouvelle-France pour ensuite faire le point sur les reconstructions des «Petits Âges glaciaires» dans l'hémisphère Nord. Par la suite, nous évaluons les reconstructions à partir des données indirectes pour l'Amérique du Nord pendant la période qui nous concerne. Enfin, nous examinons les textes contemporains pour reconstituer les interprétations du climat pendant la période de la Nouvelle-France. Les perspectives coloniales, aussi subjectives soient-elles, concordent jusqu'à un certain point avec la reconstruction pluridisciplinaire actuelle du climat du XVIII ${ }^{\mathrm{e}}$ siècle. Toutefois, les concepts de causalité diffèrent de ceux proposés aujourd'hui.

\section{L'HISTOIRE DU CLIMAT}

Seuls quelques historiens se sont penchés sur l'histoire climatique de la Nouvelle-France. Deux articles des années 1970 ont analysé les descriptions du climat faites par les Jésuites et par le voyageur finnois Pehr Kalm. La biogéographe Louise Filion a présenté un survol des acquis dendrochronologiques pour proposer un cadre climatique pluriséculaire pour le Québec. Pour sa part, Thomas Wien a pris en compte les fluctuations climatiques pour établir le calendrier agricole qui encadrait les activités économiques des habitants 5 . Plus récemment, dans sa comparaison de l'Acadie avec le Loudunais, Gregory Kennedy a commenté l'impact du Petit Âge glaciaire sur les deux régions à la fin du XVII et au début du XVIII ${ }^{e}$ siècle: "a period of greater climatic variability and especially cold winters in both places». Si la population du Loudunais était plus affectée par les variations climatiques jusqu'au début du XVIII ${ }^{e}$ siècle, les deux régions ont bénéficié de la croissance démographique et économique pendant la période climatique plus clémente entre 1710 et $1740^{6}$.

5. Richard Arès, "Les Relations des Jésuites et le climat de la Nouvelle-France», Délibérations et Mémoires de la Société royale du Canada (quatrième série, tome VIII, 1970): 75-91; Benoît Brouillette, "Quelques observations climatiques en Nouvelle-France au dix-huitième siècle », dans ibid., 93-99; Louise Filion, «Le cadre climatique au Québec pendant la période historique», dans Philip P. Boucher, dir., Actes du onzième colloque de la French Colonial Historical Society, Québec, mai 1985 (Lanham, University Press of America, 1987): 73-79; Thomas Wien, "Les travaux pressants". Calendrier agricole, assolement et productivité au Canada au XVIII" siècle", Revue d'histoire de l'Amérique française, 43, 4 (1990): 535-558.

6. Gregory Kennedy, Something of a Peasant Paradise? Comparing Rural Societies in Acadie and the Loudunais, 1604-1755 (Montréal, McGill-Queen's University Press, 2014), 29. 
Pour sa part, Victoria Slonosky a étudié les statistiques météorologiques colligées par le médecin Jean-François Gaultier entre 1742 et 1756 pour situer ses observations dans une chronologie plus longue. Elle compare les données de Gaultier avec celles prises dans les forts de la Compagnie de la Baie d'Hudson. Si certains mouvements de température se rapprochent de ceux enregistrés plus au nord, observe l'auteure, d'autres demeurent distincts. Étant donné les distances entre les forts de la Baie d'Hudson et Québec, il n'est pas surprenant que les expériences aient divergé. De plus, les températures ont continué de varier: les périodes plus froides sont aussi possibles dans une époque par ailleurs plus modérée ${ }^{7}$. Ces contributions importantes démontrent déjà l'importance du climat pour une société qui se basera sur une économie largement agricole.

Notre objectif est un peu différent des approches de ces chercheurs. Nous voulons établir ici le lien entre la compréhension locale du climat et les changements climatiques durant la période du Petit Âge glaciaire. D’abord, commençons avec la définition du climat considéré d'un point de vue historique. Du point de vue scientifique, le climat représente le total des événements météorologiques - qu'ils soient de nature extrême ou plutôt habituelle - pendant une période d'au moins une décennie, dans un endroit défini, qui peut être local ou même global.

Plusieurs historiens du climat ont adopté cette définition, mais ils mettent l'accent sur des éléments différents concernant la compréhension du passé climatique. Pour certains, il faut situer le changement climatique à l'intérieur des tendances de longue durée. D’autres s'intéressent plutôt aux événements météorologiques individuels, qu'ils conçoivent soit comme des charnières du changement graduel du climat, ou tout simplement comme les manifestations des tendances climatiques générales. Certains historiens du climat traversent les échelles géographiques et temporelles afin de faire le lien entre les développements climatiques globaux et les expériences locales que les peuples ont vécues. Finalement, d'autres insistent sur le fait que le climat est un construit social reflétant le contexte politique, social ou scientifique. Dans ce texte, nous intégrons toutes ces approches différentes à l'étude du climat historique de la Nouvelle-France ${ }^{8}$.

7. Victoria C. Slonosky, «The Meteorological Observations of Jean-François Gaultier, Quebec, Canada: 1742-56», Journal of Climate, 16 (juillet 2003): 2232-2247; Victoria Slonosky, «Daily minimum and maximum temperature in the St-Lawrence Valley, Quebec: two centuries of climatic observations from Canada ", International Journal of Climatology (2014), http://dx.doi.org/10.1175/1520-0442 (2003) $16<2232$ :TMOOJG > 2.0.CO ;2.

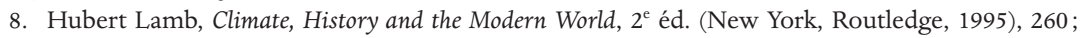
Lenny Bernstein et al., Climate Change 2007: Synthesis Report, An Assessment of the Intergovernmental Panel 
C'est à l'aide d'une multitude de sources que les chercheurs contribuent à la reconstruction pluridisciplinaire des changements climatiques. Les scientifiques reconstruisent les climats d'antan en utilisant des sources paléoclimatiques diverses: des entités matérielles qui répondent à la fluctuation climatique et qui peuvent parfois se substituer aux mesures instrumentales. Ces données indirectes proviennent des carottes de glace, des cernes, des sédiments des lacs, des débris planctoniques, des tourbières et d'autres archives «naturelles» de l'hydrosphère, de la biosphère et de la cryosphère (les endroits où l'eau est gelée). La reconstruction des climats prend en compte aussi l'avancement ou le recul des lignes d'arbres, des glaciers et la répartition des espèces, qui répondent de façon prévisible aux tendances climatiques, et qui jumelés avec d'autres données indirectes peuvent aider à développer des modèles de la variabilité des climats du passé. L'archéologie et, pour des périodes plus récentes, la recherche dans les textes historiques peuvent renforcer la précision des résultats scientifiques ${ }^{9}$.

\section{LES PETITS ÂGES GLACIAIRES}

Le consensus actuel suggère que les températures moyennes partout dans l'hémisphère Nord et dans d'autres parties du monde se sont refroidies entre le milieu du XIII ${ }^{\mathrm{e}}$ siècle et le milieu du XIX ${ }^{\mathrm{e}}$, par rapport aux normes du début du $\mathrm{XX}^{\mathrm{e}}$ siècle. Ce «Petit Âge glaciaire» représente peut-être l'anomalie climatique la plus significative des 8000 dernières années, jusqu'au réchauffement actuel de la planète. Mais l'idée d'un Petit Âge glaciaire n'implique pas qu'il faisait toujours froid d'année en année, et de saison en saison. Des années et même des décennies plutôt chaudes ont souvent interrompu les périodes plus froides.

Le Petit Âge glaciaire peut être défini comme une ère aussi bien de variabilité que de temps froid. Il vaudrait peut-être mieux distinguer des épisodes spécifiques, de "vrais petits âges glaciaires», qui ont prévalu pendant moins d'un siècle sur une partie très étendue de la Terre. Or même ces périodes relativement froides étaient moins marquées par des constantes météorologiques que par des mouvements extrêmes plus fréquents. À la base de ces fluctuations se trouvent des variations dans l'or-

on Climate Change (Valence, IPCC, 2007), 30; Mark Carey et Philip Garone, "Forum Introduction», Environmental History, 19 (2014): 285.

9. Rudolf Brázdil et al., "Historical Climatology in Europe - the State of the Art», Climatic Change 70, 3 (2005): 364 ; Joëlle Gergis et al., "The Influence of Climate on the First European Settlement of Australia: A Comparison of Weather Journals, Documentary Data and Palaeoclimate Records, 17881793 ", Environmental History, 15, 3 (2010): 504; Neville Brown, History and Climate Change: A Eurocentric perspective (Londres, Routledge, 2001), 315 ; H. Lamb, Climate, History and the Modern World, op. cit., 148. 
bite de la Terre et dans la radiation solaire. Plus significative, une série d'éruptions volcaniques a émis des aérosols dans l'atmosphère, qui eurent pour effet de disperser la lumière du soleil et ainsi de refroidir la troposphère (l'atmosphère entre 8 à 15 kilomètres d'altitude), ce qui perturba la cryosphère. Le résultat fut des réductions des températures à long terme partout dans le monde ${ }^{10}$.

Le XVII ${ }^{\mathrm{e}}$ siècle, qui fut le plus froid du Petit Âge glaciaire, a connu deux phases parmi les plus froides de la période moderne. Selon des données scientifiques indirectes et textuelles, à la fin du XVI siècle, les températures annuelles moyennes ont décliné presque partout dans l'hémisphère Nord, comme en Afrique, en Amérique du Sud et en Asie. Le fait de l'expansion contemporaine du glacier voisin de la commune suisse de Grindelwald inspire le terme la «Fluctuation de Grindelwald» pour décrire la période qui va de 1565 à 1628, environ. Dans plusieurs endroits de l'hémisphère Nord, les températures saisonnières étaient alors plus froides d'au moins un degré Celsius au-dessous des moyennes du XX $\mathrm{X}^{\mathrm{e}}$ siècle. Les interactions sont complexes: des températures moyennes plus basses et les différentes parties de l'atmosphère, de l'hydrosphère, de la biosphère et de la cryosphère ont créé des changements régionaux complexes non seulement du régime climatique «normal», mais aussi des courants océaniques, de la distribution de la glace de mer et d'autres conditions environnementales. Précisons que l'impact local différait beaucoup selon la région et que certaines régions ne semblaient pas ressentir l'impact de la Fluctuation de Grindelwald ${ }^{11}$.

10. Sam White, "The Real Little Ice Age», The Journal of Interdisciplinary History, 44, 3 (hiver, 2014): 327; Shaun A. Marcott et al., "A Reconstruction of Regional and Global Temperature for the Past 11,300 Years", Science, 339, 6124 (2013): 1198; Jean M. Grove, Little Ice Ages: Ancient and Modern (Londres, Routledge, 2004), 2; Michael E. Mann, "The Little Ice Age», dans Ted Munn, dir., Encyclopedia of Global Environmental Change (Chicester: John Wiley \& Sons, 2002), 1256; Hubert Lamb, «Volcanic Dust in the Atmosphere; with a Chronology and Assessment of its Meteorological Significance», Philosophical Transactions of the Royal Society of London, Series A: Mathematical and Physical Sciences, 266, 1178 (1970): 462; K. Briffa, P. D. Jones, F. H. Schweingruber, et T. J. Osborn, «Influence of volcanic eruptions on Northern Hemisphere summer temperature over the past 600 years", Nature, 393 (1998): 451; Gifford Miller et al., "Abrupt onset of the Little Ice Age triggered by volcanism and sustained by sea-ice/ ocean feedbacks», Geophysical Research Letters, 39, 2 (2012): 4; Y. Zhong et al., "Centennial-scale climate change from decadally-paced explosive volcanism: a coupled sea ice-ocean mechanism», Climate Dynamics, 37 (2011): 2373; Alan Robock, "Volcanic Eruptions and Climate» Reviews of Geophysics, 38, 2 (mai 2000): 194.

11. K. R. Briffa et al., "European Tree Rings and Climate in the 16th Century", Climatic Change, 43, 1 (1999): 166; R. Neukom et al., «Multiproxy summer and winter surface air temperature field reconstructions for southern South America covering the past centuries ", Climate Dynamics, 37, 1-2 (2011): 42; S. Ge et al., "Temperature variation through 2000 years in China: An uncertainty analysis of reconstruction and regional difference», Geophysical Research Letters, 37 (2010): 4 ; Fredrik Charpentier Ljungqvist, "A New Reconstruction of Temperature Variability in the Extra-Tropical Northern Hemisphere During the Last Two Millennia », Geografiska Annaler, 92, 3 (2010): 345. 
Au milieu du XVII ${ }^{e}$ siècle, les températures moyennes dans plusieurs endroits ont généralement augmenté selon des chronologies et des graduations diverses. Cependant, vers la fin du siècle, les températures ont ensuite chuté de façon marquée, une reprise en quelque sorte du déclin associé à la Fluctuation de Grindelwald. Cette nouvelle période, le "Minimum de Maunder» (entre 1660 et 1720 environ), représente la deuxième phase froide du Petit Âge glaciaire. L'astronome Edward Maunder, qui a identifié la réduction subite des taches solaires vers la fin du XVII siècle, a prêté son nom au phénomène. Dans plusieurs régions, la dernière décennie du XVII ${ }^{\mathrm{e}}$ siècle était extrêmement glaciale. Par la suite, les températures moyennes annuelles ont subi une hausse au XVIII siècle. Une fois de plus, ces changements dans les températures moyennes ont déclenché des réponses météorologiques et environnementales complexes et parfois contraires à l'intuition ${ }^{12}$.

L'historien Fredrik Charpentier Ljungqvist a créé la courbe suivante pour démontrer les fluctuations climatiques depuis l'an 1 de notre ère. La Fluctuation de Grindelwald et le Minimum de Maunder apparaissent clairement sur le graphique de la page suivante.

\section{RECONSTRUCTIONS DES CLIMATS EUROPÉEN ET NORD-AMÉRICAIN}

Les chercheurs européens ont été les pionniers des études sur le climat historique, rendant les reconstructions des températures moyennes européennes pendant la Fluctuation de Grindelwald et le Minimum de Maunder particulièrement fiables. Selon ces chercheurs, durant les décennies les plus glaciales des deux phases, les températures annuelles moyennes ont baissé de plus d'un degré Celsius presque partout en Europe. En outre, les historiens et les scientifiques ont lié les fluctuations des températures moyennes aux changements d'autres conditions météorologiques. Par exemple, si les températures plus froides réduisent généralement le taux d'évaporation, la précipitation et l'humidité de surface moyenne ont plutôt baissé dans plusieurs régions européennes au cours des phases les plus froides du Petit Âge glaciaire, surtout sur les côtes méditerranéennes. Par contre, la précipitation et l'humidité de surface ont augmenté dans l'Europe du Nord-Ouest, particulièrement au printemps et en automne. En même temps, le glissement

12. John Eddy, «The Maunder Minimum», Science, 192, 4245 (1976): 1189; Jürg Luterbacher, «The Late Maunder Minimum (1675-1715) - Climax of the "Little Ice Age" in Europe", dans P. D. Jones, dir., History and Climate: Memories of the Future? (New York: Kluwer Academic/Plenum Publishers, 2001), 30 ; Michael E. Mann et al., "Global signatures and dynamical origins of the Little Ice Age and Medieval Climate Anomaly", Science, 326, 5957 (novembre 2009) : 1257. 


\section{Graphique}

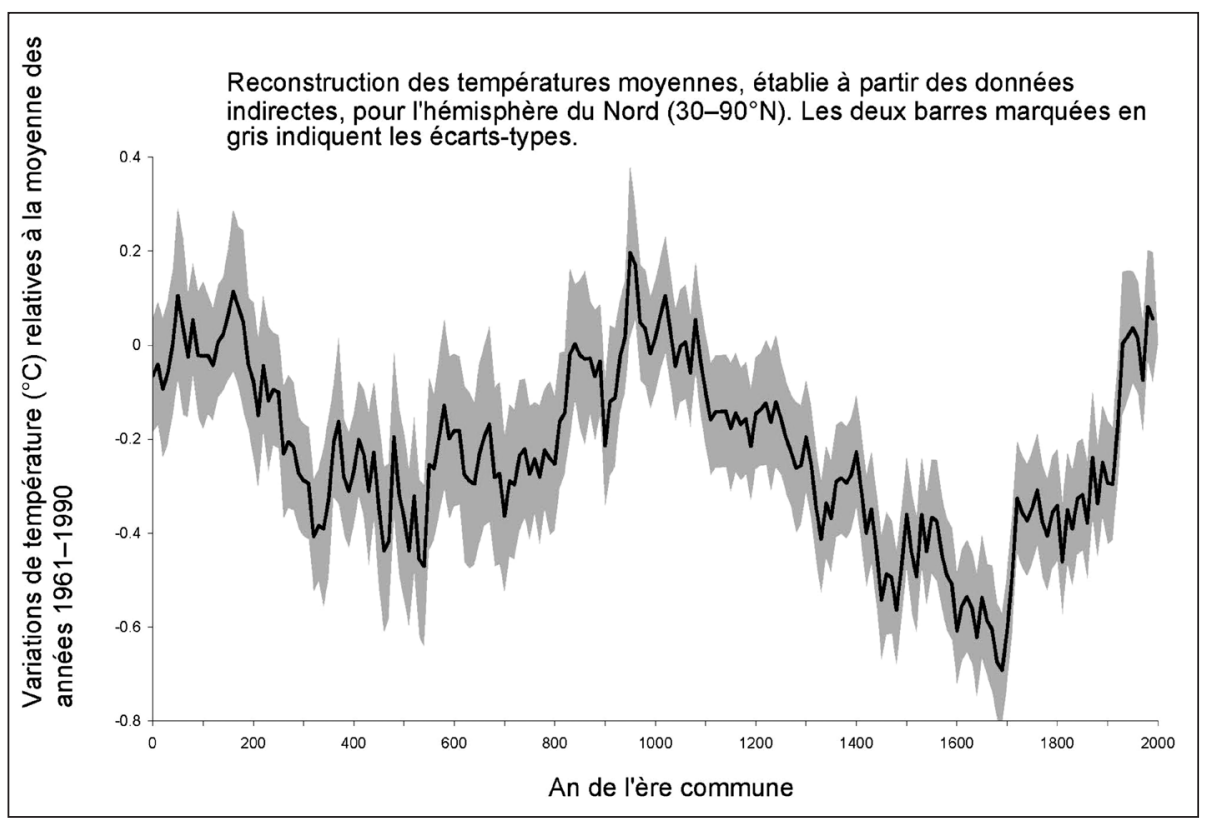

Source: Fredrik Charpentier Ljungqvist, «A New Reconstruction of Temperature Variability in the Extra-tropical Northern Hemisphere during the last Two Millenia », 345. Reproduit avec la permission du professeur Ljunqvist.

vers le sud du vortex polaire arctique, un cyclone tenace, a augmenté le nombre et l'ampleur des tempêtes dans plusieurs parties de l'Europe. En particulier, la fréquence des tempêtes s'est accrue de façon spectaculaire dans le nord-ouest. Les vents d'ouest sont devenus moins communs au cours du Minimum de Maunder à la suite des changements dans la température de la surface de la mer qui accompagnent les phases froides de l'Oscillation atlantique multidécennale, et des changements dans la pression atmosphérique nord-atlantique associés aux effets de l'Oscillation nord-atlantique ${ }^{13}$.

13. Christian Pfister et Rudolf Brázdil, «Climatic Variability in Sixteenth-Century Europe and its Social Dimension: A Synthesis», Climatic Change, 43, 1 (1999): 32 ; Christoph C. Raible, "Climate variability - observations, reconstructions, and model simulations for the Atlantic-European and Alpine region from 1500-2100 AD », Climatic Change, 79, 1-2 (2006) : 19; Rudolf Brázdil et al., "Historical Climatology in Europe - the State of the Art», op. cit., 404; Dennis Wheeler, «British Naval Logbooks from the Late Seventeenth Century: New climatic information from old sources », History of Meteorology, 2 (2005): 142; H. Lamb, Climate, History, and the Modern World..., op. cit., 231; Rüdiger Glaser et Dirk Riemann, "A 
Ces changements climatiques eurent des impacts importants sur la vie humaine. Par exemple, certains historiens ont soutenu que pendant les années les plus froides de la Fluctuation de Grindelwald, la saison agricole dans plusieurs parties de l'Europe a été raccourcie jusqu'à six semaines. Plusieurs cultivateurs ont par la suite abandonné le blé, qui croît dans des conditions chaudes et sèches, pour les cultures de l'orge, du seigle ou de l'avoine, plus rentables dans ces conditions nouvelles. Les températures plus froides, les changements dans la précipitation et le temps plutôt difficile à prédire ont accentué les problèmes des économies agraires, contribuant ainsi aux famines, qui, elles, ont été aggravées par les demandes et les privations dues aux guerres presque continuelles.

Les conditions climatiques eurent aussi des effets culturels: des communautés ont blâmé les sorcières pour ces temps destructeurs et la persécution de ces femmes a alors augmenté. Au cours de la Fluctuation de Grindelwald et du Minimum de Maunder, des températures plus froides et des modifications dans les étages de végétation dans l'Europe du Nord ont compliqué l'élevage: plusieurs éleveurs commencèrent à garder des moutons plutôt que des bœufs. Des loups se sont insérés dans les villages pendant des hivers froids, tandis que les vautours ont quitté les Alpes trop glaciales. La Tamise, la Seine et d'autres rivières européennes ont gelé complètement durant les hivers les plus froids de la période, ce qui a mis fin à la circulation sur l'eau et en même temps a permis la tenue de foires sur la glace. En fait, le changement climatique n'a pas eu que des effets destructeurs. Plusieurs Hollandais, par exemple, ont bénéficié d'une économie en plein essor au XVII ${ }^{\mathrm{e}}$ siècle. Certains Européens lettrés ont bien noté le fait que les événements extrêmes sont devenus plus communs pendant la Fluctuation de Grindelwald et le Minimum de Maunder que dans les décennies antérieures ${ }^{14}$.

Les reconstructions du climat historique nord-américain indiquent que les tendances se distinguèrent passablement du refroidissement européen

thousand-year record of temperature variations for Germany and Central Europe based on documentary data ", Journal of Quaternary Science, 24, 5 (2009): 445 ; D. Wheeler et al., "Atmospheric circulation and storminess derived from Royal Navy logbooks: 1685 to 1750 ", Climatic Change, 101, 1-2 (2010): 257; Dagomar Degroot, " "Never such weather known in these seas:" Climatic Fluctuations and the AngloDutch Wars of the Seventeenth Century, 1652-1674", Environment and History, 20, 2 (mai 2014): 239-273. J. Luterbacher et al., «Extending North Atlantic Oscillation reconstructions back to 1500 », Atmospheric Science Letters, 2, 1-4 (juin 2002): 114-124.

14. Wolfgang Behringer, A Cultural History of Climate (Cambridge, Polity Press, 2010), 117; Dagomar Degroot, The Frigid Golden Age: Experiencing Climate Change in the Dutch Republic, 1560-1720, thèse de doctorat (histoire), Université York, 2014, 469; Geoffrey Parker, Global Crisis: War, Climate Change and Catastrophe in the Seventeenth Century (New Haven, Yale University Press, 2013), 56. 
du XVII ${ }^{\mathrm{e}}$ siècle. Mais en Amérique aussi la diversité s'imposait d'une région à l'autre. Par exemple, la dendrochronologie établie par des chercheurs à partir de la ligne d'arbres en Alaska et dans les territoires canadiens affirme que le Grand Nord a connu la Fluctuation de Grindelwald ainsi que le Minimum de Maunder. À l'ouest du continent, les températures étaient relativement froides au XVII ${ }^{e}$ siècle, même si les preuves ne permettent pas de démarquer les deux "vrais petits âges glaciaires». Par contre, selon des sources concernant ce qui est maintenant le nord-est des États-Unis, le refroidissement du XVII ${ }^{e}$ siècle n'a laissé que peu de traces. Des reconstructions des températures de la surface de la mer d'après des techniques paléoclimatiques révèlent que la baie de Chesapeake était relativement chaude pendant la majorité du XVII siècle et plus froide de façon marquée au XVIII ${ }^{e}$ siècle. Les analyses scientifiques des sédiments des lacs étudiés ont démontré que les glaciers se sont avancés dans les Rocheuses canadiennes non pas au XVII siècle mais plutôt au début du $\mathrm{XVIII}^{\mathrm{e}}$ siècle, à la suite de précipitations régionales plus importantes ${ }^{15}$.

Tout comme en Europe, des changements dans les tendances des précipitations en Amérique du Nord ont coïncidé avec des mouvements dans les températures moyennes. Par exemple, des sécheresses ont affligé la Nouvelle-Espagne de façon systématique au cours de la Fluctuation de Grindelwald, tandis que le sud-est du continent était extraordinairement humide pendant la Fluctuation de Grindelwald et le Minimum de Maunder. Certes, des saisons extrêmes, tel l'hiver glacial de 1557-1558, eurent un impact sur les deux côtés de l'Atlantique. À travers le continent, les températures ont baissé de manière plus marquée dans la première moitié du XIX ${ }^{\mathrm{e}}$ siècle qu'au XVII siècle. Sur les deux continents, le XVIII ${ }^{\mathrm{e}}$ siècle fut généralement plus doux que les siècles antérieurs et subséquents ${ }^{16}$.

En comparaison avec l'historiographie du climat européen, les historiens ont peu commenté la relation entre le changement climatique de

15. R. D. D’Arrigo et G. C. Jacoby Jr., "Dendroclimatic evidence from northern North America», dans Raymong S. Bradley et Philip D. Jones, dir., Climate since A.D. 1500 (New York, Routledge, 2003), 302 ; M. E. Mann, "The Little Ice Age», dans T. Munn, dir., Encyclopedia, op. cit., 1259; John P. Cropper et Harold C. Fritts, "Tree-ring Width Chronologies from the North American Arctic», Arctic and Alpine Research, 13, 2 (1981): 249; H. C. Fritts et X. M. Shao, «Mapping climate using tree-rings from western North America», dans R. S. Bradley et P. D. Jones, dir., Climate since A.D. 1500, op. cit., 279; W. R. Baron, "Historical climate records from the northeastern United States, 1640 to 1900 », dans ibid., 83 ; T. M. Cronin et al., "Medieval Warm Period, Little Ice Age and 20th century temperature variability from Chesapeake Bay", Global and Planetary Change, 36, 1-2 (2003): 17.

16. G. Parker, Global Crisis, op. cit., 6; David W. Stahle et Malcolm K. Cleaveland, "Tree-ring Reconstructed Rainfall over the Southeastern U.S.A. During the Medieval Warm Period and Little Ice Age », Climatic Change, 26, 2-3 (1994): 206. 
l'époque moderne et l'histoire de l'Amérique du Nord. Certains ont tenté d'attribuer les épreuves des premiers colons anglais à l'influence du Petit Âge glaciaire, même si des reconstructions pluridisciplinaires du climat ont contredit leurs arguments. Plus récemment, des historiens ont interpreté les voyages d'exploration dans l'Arctique canadien, la propagation de la variole dans les Plaines, l'expansion des glaciers dans la Chaîne SaintÉlie entre l'Alaska et le Yukon, et les économies agricoles s'étendant du Nouveau-Brunswick jusqu'à la Nouvelle-Espagne selon l'optique des périodes froides du Petit Âge glaciaire et leurs expressions météorologiques diverses ${ }^{17}$.

Malgré ces contributions importantes, nous avons relativement peu d'indications qui permettent de mesurer l'impact du Petit Âge glaciaire sur la partie septentrionale de l'Amérique du Nord. Certains documents du XVII ${ }^{e}$ siècle donnent l'impression que les habitants de la vallée du Saint-Laurent ont connu une histoire climatique assez similaire à celle des habitants des colonies hollandaise et anglaises situées au sud. Ceux-ci ont probablement expérimenté un XVII ${ }^{\mathrm{e}}$ siècle relativement doux. Vers le milieu du siècle, les Jésuites et d'autres observateurs dans la vallée du Saint-Laurent ont remarqué onze hivers particulièrement froids et neuf hivers anormalement chauds. Ils ont décrit des hivers d'une durée moyenne de 150 jours, chiffre similaire aux normes connues vers le milieu du XXe siècle. En outre, les mentions assez rares des ponts de glace entre Québec et Lévis indiquent que les hivers dans cette région entre 1620 et 1740 étaient relativement doux. Néanmoins, des problèmes majeurs affectent cette interprétation des sources concernant les hivers de la vallée du Saint-Laurent. La plupart des sources ont été écrites vers le milieu du XVII ${ }^{\mathrm{e}}$ siècle, après le nadir de la Fluctuation de Grindelwald et avant l'impact du Minimum de Maunder sur l'hémisphère Nord. De plus, le gel complet du fleuve qui permet la formation des ponts de glace n'avait pas nécessairement comme origine des hivers globalement froids mais plutôt des vagues de froid brusques en interaction complexe avec la morphologie

17. Karen Ordahl Kupperman, "The Puzzle of the American Climate in the Early Colonial Period», American Historical Review, 87, 5 (1982): 1264; Adam R. Hodge, "In Want of Nourishment for to Keep Them Alive" : Climatic Fluctuations, Bison Scarcity, and the Smallpox Epidemic of 1780-82 on the Northern Great Plains», Environmental History, 17, 2 (2012): 400; Liza Piper, "Colloquial Meteorology», dans Alan MacEachern et William J. Turkel, dir., Method and Meaning in Canadian Environmental History (Toronto, Nelson Education, 2009), 117; Dagomar Degroot, «Exploring the North in a Changing Climate: The Little Ice Age and the Journals of Henry Hudson, 1607-1611", Journal of Northern Studies (à paraître); Bradley Skopyk, Undercurrents to Conquest: The Shifting Terrain of Indigenous Agriculture in Colonial Tlaxcala, Mexico, thèse de doctorat (histoire), Université York, 2010, 10; Julie Cruikshank, Do Glaciers Listen? Local Knowledge, Colonial Encounters, and Social Imagination (Vancouver, UBC Press, 2005), 24. 
du fleuve. Enfin, les documents, souvent écrits à la fin de l'année, ont tendance à mettre l'hiver beaucoup plus en évidence que les autres saisons. Il est donc difficile, à partir des seules sources documentaires, de reconstruire le climat de façon sûre ${ }^{18}$.

Pour les régions plus au nord, l'examen dendrochronologique des arbres du Nouveau-Québec suggère une histoire climatique quelque peu différente. On observe, en effet, que les arbres du Nouveau-Québec ont à peine crû pendant la Fluctuation de Grindelwald et le Minimum de Maunder. Selon des experts scientifiques, ces périodes de faible croissance sont en relation directe avec les hivers glacials et venteux avec peu de neige, et des étés froids et humides. Ces conclusions appuient l'étude des dépôts de pollen extraits des fonds de lacs dans le sud-ouest du Québec, ce qui indique un refroidissement important à la fin du XVI et au début du XVII ${ }^{e}$ siècle. Les colons français dans la vallée du Saint-Laurent ont-ils connu un $\mathrm{XVII}^{\mathrm{e}}$ siècle relativement doux tel qu'il est présenté dans les documents, ou ont-ils plutôt expérimenté l'histoire climatique plus froide que proposent ces données indirectes? Puisque les sources scientifiques bénéficient de plus de continuité et peuvent être analysées de façon plus systématique pour cette région, il nous est permis de conclure que dans la vallée du Saint-Laurent, le XVII siècle était généralement plus froid que la première moitié du XVIII ${ }^{e}$ siècle. Au XVII ${ }^{e}$ siècle, les habitants de la vallée du Saint-Laurent expérimentèrent les années très froides qui ont affecté tout l'hémisphère Nord. Le Minimum de Maunder a, en effet, bel et bien frappé la Nouvelle-France ${ }^{19}$.

\section{LE CONCEPT DU CLIMAT AU XVII ET AU XVIII ${ }^{e}$ SIÈCLE}

Ayant tracé les grandes lignes de l'histoire du climat en Europe et en Nouvelle-France aux XVII ${ }^{e}$ et XVIII ${ }^{e}$ siècles, nous allons maintenant aborder les interprétations contemporaines de ces phénomènes. D’abord,

18. Norman Clermont, "A-t-on vécu les hivers d'un petit âge glaciaire en Nouvelle-France?», Géographie physique et Quaternaire, 50, 3 (1996): 396; Daniel Houle et Jean-David Moore, «Les ponts de glace sur le fleuve Saint-Laurent. Un indicateur de la sévérité des hivers entre 1620 et 1910 », Le Naturaliste Canadien, 132, 1 (hiver, 2008): 76.

19. Serge Payette et al., "Secular climate change in old-growth tree-line vegetation of northern Quebec», Nature, 315, 135-138, (1985): 137; Claude Lavoie et Serge Payette, «Black Spruce Growth Forms as a Record of a Changing Winter Environment at Treeline, Quebec, Canada ", Arctic and Alpine Research, 24, 1 (1992): 48; Gordon C. Jacoby, Ileana S. Ivanciu et Linda D. Ulan, «A 263-year record of summer temperature for northern Quebec reconstructed from tree-ring data and evidence for a major climatic shift in the early 1800’s ", Palaeogeography, Palaeoclimatology, Palaeoecology, 64 (1988): 75 ; Nathalie Paquette et Konrad Gajewski, "Climatic change causes abrupt changes in forest composition, inferred from a high-resolution pollen record, southwestern Quebec, Canada », Quaternary Science Reviews, 75 (2013): 169. 
il faut reconnaître que jadis le mot "climat» possédait un sens plus large que celui que nous lui accordons aujourd'hui. Dans la pensée d'Aristote, le climat représente un phénomène beaucoup plus vaste que la simple météorologie. Il faut prendre en compte la latitude, l'altitude et toutes les conditions qui affectent la vie humaine. Pendant l'époque moderne, les savants croyaient que le climat déterminait le caractère national d'un peuple. Pour les Européens, les théories climatiques ont renforcé leurs idées de supériorité ${ }^{20}$.

Le climat gouvernait la vie des individus dans la colonie lointaine. Le «climat» influait énormément sur la santé des habitants. Comme Stéphanie Tésio l'a démontré, des médecins comme Jean-François Gaultier attribuaient plusieurs maladies aux changements de température. Par exemple, le froid hivernal, selon le médecin, était à l’origine des pleurésies et des péripneumonies. Gaultier envoya ses observations scientifiques quotidiennes de la température en parallèle avec ses commentaires sur la santé des habitants de la colonie. Pour lier climat et maladies, il se basait sur les théories d'Hippocrate, rejettant explicitement la théorie miasmatique ${ }^{21}$.

Le climat avait aussi des résonances populaires, car tout habitant de la colonie devait le prendre en considération. Pour une population pré-industrielle, le climat représente une réalité à laquelle les habitants devaient faire face tous les jours de façon expérientielle. Nul ne pouvait ignorer les différences réelles entre le climat français et celui du Nouveau Monde septentrional. Dans une colonie qui allait devenir rapidement agricole, reconnaître les mouvements des saisons indiquait que l'on se préparait adéquatement pour produire des surplus agricoles. Thomas Wien a expliqué comment les habitants de la Nouvelle-France se sont créés un calendrier agricole qui reconnaissait les limites climatiques de la colonie: «Au Canada, une vieille agriculture paysanne, forte de ses réflexes familiaux et de ses gestes enchevêtrés, rencontre un climat sévère. » Ils s'efforcent de terminer la fenaison et de récolter du blé avant le premier gel de l'automne. Les habitants du sud du Québec ne disposaient que de 130 à 150 jours environ de la semence jusqu'à la récolte ${ }^{22}$. En effet, le succès de l'économie familiale dépendait du savoir climatique de l'habitant. Si les agriculteurs n'écrivaient pas de jour-

20. Clarence J. Glacken, Traces on the Rhodian Shore: Nature and Culture in Western Thought from Ancient Times to the End of the Eighteenth Century (Berkeley, University of California Press, 1967), 451; Fabien Locher et Jean-Baptiste Fressoz, «Modernity's Frail Climate: A Climate History of Environmental Reflexivity", Critical Inquiry, 38 (printemps 2012): 587.

21. Stéphanie Tésio, "Climat et médicine à Québec au milieu du $18^{\mathrm{e}}$ siècle ", Scientia Canadensis, 31 , 1-2 (2008): 155-165.

22. T. Wien, "Les travaux pressants”...", loc. cit., 545. 
naux intimes avec des refléxions journalières sur le temps et les températures, comme ce fut le cas chez certains agriculteurs anglophones au XIX ${ }^{\mathrm{e}}$ siècle $e^{23}$, ils savaient bien comment le climat dans la colonie pouvait fluctuer. Même pendant les décennies plus douces au XVIII ${ }^{e}$ siècle, des disettes eurent lieu en 1736-1737 et 1741-1743. Ces événements furent aggravés par la longue période entre le début de l'hiver et la récolte de l'année subséquente ${ }^{24}$. La marge de manœuvre était relativement restreinte, étant donné les caprices du temps. Alors, comme Gaultier le remarqua pour l'année 1744 , lorsque le temps permit des semences précoces, toute la colonie en bénéficia: "parce qu'elles sont en état d'être recueillies vers la mi-Août, $\&$ par ce moyen-là elles sont garanties des coups de Soleil qui les échaudent, $\&$ des pluies abondantes qui font presque autant de tort à la récolte que les grandes chaleurs ${ }^{25}$ \%.

\section{DÉCRIRE LE CLIMAT DE LA NOUVELLE-FRANCE}

Des descriptions détaillées comme celles de Gaultier sont rarissimes. Comme toute la population faisait face à la même expérience climatique, peu de raisons justifiaient de la décrire localement. Néanmoins, il était souvent approprié d'expliquer le climat de façon générale à des lecteurs européens. Alors, il fallait décrire les conditions en faisant référence explicitement à l'expérience française. Les écrits s'efforcèrent d'expliquer les hivers - et les étés - en soulignant leurs différences avec ceux de la France. C'est surtout le grand froid de l'hiver canadien qui surprend les observateurs français, mais les étés parfois très chauds et humides soulèvent aussi des questions. Les observateurs s'étonnaient surtout de ce qu'on appellerait aujourd'hui le climat continental: les étés chauds et des hivers très froids, accentués par les vents venant soit du golfe du Mexique ou de l'Arctique. Mais ils trouvaient aussi d'autres facteurs pour expliquer les contrastes entre le Canada et la France.

Dans sa Relation publiée en 1616, le jésuite Pierre Biard commençait sa description du climat de la Nouvelle-France en faisant référence à l'influence «satanique» qui distinguait la mère patrie de l'Amérique septentrionale. La Nouvelle-France devait être une «terre jumelle avec la nostre, subjecte à mesmes influences, rangée en mesme parallele, située en

23. L. Piper, "Colloquial Meteorology... », loc. cit., 102-123.

24. Louise Dechêne, Le Partage des subsistances au Canada sous le régime français (Montréal, Boréal, 1994), 98-102.

25. H. L. duHamel du Monceau, «Observations botanicos-météorologiques faites à Québec par M. Gaultier pendant... l'année 1744 ", Mémoires de l'Académie Royale des Sciences (1745): 214-215. 
mesme climat». Cependant, la "malice de Satan» la rend désagréable, c'est-à-dire distincte de la mère patrie. Pour lui, comme pour bien d'autres observateurs, la différence fut quelque peu surprenante, particulièrement le grand froid d'hiver:

Ces terres estant, come nous avons dit, paralleles à nostre France, c'est à dire, en mesme climat, $\&$ mesme elevation, par reigle d'Astrologie, elle doivent avoir mesme influences, mesmes inclinations, $\&$ temperatures... [Q]uoy qu'en disent les Astrologues, si faut-il advouër que ce païs là (parlant universellement, $\&$ comme il est à ceste heure) est plus froid que n'est nostre France, $\&$ qu'il y a diversité grande quant aux temps $\&$ saisons de l'un à l'autre ${ }^{26}$.

Les descriptions climatiques prirent en compte la latitude, tout en exposant les différences.

Au tournant du XVIII siècle, le jésuite Louis Nicolas dut expliquer dans son manuscrit sur l'histoire naturelle de la Nouvelle-France, écrit à l'intention du roi et des membres de sa cour, que les hivers distinguaient la colonie de la France. L'hiver représentait « cette saison extrêmement froide dans le climat du Nouveau Monde septentrional et occidental, quoiqu'il soit à peu près sous les mêmes élévations de notre France ${ }^{27}$ ». Le père Charlevoix commenta les hivers extrêmes de la colonie: «L'Hyver... commence toujours de maniere à étonner quiconque n'y est pas fait. » Il nota aussi la chaleur de l'été, pour poser la question: «D”où peut donc venir cette différence de Température sous les mêmes Paralleles ${ }^{28}$ ?" Jusqu'à la fin du Régime français, les observateurs comparèrent les latitudes de la Nouvelle et de l'ancienne France. Après une carrière dans l'administration coloniale, Nicolas-Gaspard Boucault retourna en France et laissa une description du territoire. Il décrivit la Nouvelle-France dans les mêmes termes que ses prédécesseurs: "On a peine à croire que les hivers soient plus longs et plus rudes en ce pays qu'en France parce qu'il se trouve à peu près à la même hauteur du soleil ${ }^{29}$. » Les explications climatiques habituelles n'arrivaient pas à éclairer ce nouveau contexte géographique.

26. Reuben Gold Thwaites, dir., The Jesuit Relations and Allied Documents (Cleveland, Burrows Brothers, 1908), vol. 3 : 32, 46. Pour Claude Charles Le Roy Bacqueville de la Potherie, le climat du pays des Iroquois aurait dû aussi être moins sévère: "Si l'on ne consideroit que le Ciel, leur climat devroit être fort doux.», Histoire de l'Amérique septentrionale qui contient l'histoire des Iroquois (Paris, Nyon Fils, 1753), 3.

27. François-Marc Gagnon et al., The Codex Canadensis..., op. cit., 449.

28. P. de Charlevoix, Journal d'un voyage fait par ordre du roi dans l'Amérique septentrionale (Paris, Chez Rollin fils, 1744), 164, 167.

29. "État présent du Canada, dressé sur un nombre de mémoires et connaissances acquises sur les lieux, par le sieur Boucault (1754)", Rapport de l'archiviste de la Province de Québec (1920-1921), 23. 
Les différences entre le climat de la métropole et celui de la colonie n'étaient pas toujours perçues de façon négative. Les hivers y sont plus froids qu'en France, mais pour la même raison l'air y est plus sain. Pour le jésuite Paul Le Jeune, après la traversée difficile de l'océan, les arrivants se rétablissaient facilement: «L'air de la Nouvelle France est tres-sain pour l'ame $\&$ pour le $\operatorname{corps}^{30}$.» Quelques observateurs reconnurent les différences, mais ils essayèrent tout de même d'en minimiser l'importance. Pierre Boucher, qui écrivit son livre en 1664 pour promouvoir la colonisation française, reconnut qu'il fallait répondre aux perspectives critiques sur le climat colonial. Il expliqua que les étés n'étaient pas plus chauds que dans le pays d'Aunis, c'est-à-dire près de La Rochelle. Les hivers étaient plus froids qu'en France, convenait-il, mais on s'y habituait en Nouvelle-France: «Il y a quelques journées qui sont bien rudes... on s'habille un peu plus qu'à l'ordinaire.» D’ailleurs, puisque le bois de chauffage était facilement accessible, les habitants firent «bon feu dans les maisons». Si l'hiver prolongé imposait de nourrir le cheptel dans les granges pendant plusieurs mois, la neige servait à faciliter la mobilité. D’ailleurs, le froid avait des effets bénéfiques: «L'air est extremément sain en tout temps: mais sur tout l'Hyver; on voit rarement des maladies en ces Pays-icy. » Le climat pouvait donc contribuer à la santé de l'individu en permettant la salubrité ${ }^{31}$. De son côté, le militaire Louis-Antoine de Bougainville laissait une description relativement positive des hivers dans la colonie: «L'hiver est toujours très rude en Canada, le froid y est cependant toujours beau et fort sec, pourvu qu'il n'y ait pas du vent de nord-est ${ }^{32}$.»

Dans une colonie qui deviendra rapidement agricole, le climat plus rude n'exclut pas l'établissement, surtout lorsque les habitants s'étendaient de plus en plus vers le sud-ouest. En 1650, le jésuite Paul Ragueneau proclama que les terres dans la région de Montréal s'avéraient excellentes. Si ce n'était de l’hostilité des Iroquois, «ce lieu seroit un Paradis terrestre». Plus on avançait vers le sud, plus le climat ressemblait au sud de la France: «[dans les] quartiers plus meridionaux du Canada... la terre y porte d'elle mesme, les mesmes especes d'arbres $\&$ de fruits, que produit la Provence; aussi se trouve-t'elle sous un climat, qui a presque la mesme temperature

30. R. G. Thwaites, dir., Jesuit Relations..., op. cit., vol. 21, 108.

31. P. Boucher, Histoire véritable et naturelle, op. cit., 140-143.

32. Louis-Antoine de Bougainville, «Mémoire sur l'état de la Nouvelle-France», Rapport de l'archiviste de la Province de Québec (1923-1924): 55. La lettre de Simon Denys de 1651 est tout aussi enthousiaste concernant la salubrité de la colonie : Lucien Campeau, «Un témoignage de 1651 sur la Nouvelle-France», RHAF, 23, 4 (1970): 601-612. 
de l'air, $\&$ dont la hauteur du Pole n'est pas bien differente ${ }^{33}$.» Pour le jésuite François Joseph Le Mercier, l'établissement des familles des militaires promettait un avenir français pour cette partie de l'Amérique ${ }^{34}$.

L’ingénieur Louis Franquet, écrivant son rapport dans la décennie 1750, expliqua que le climat plus froid ne représentait point un obstacle aux rendements agricoles: «il y ait jamais eu aucune année, quelque contraire que la température des saisons ait été aux bleds et aux autres productions de la terre, où la récolte n'ait pas été assez abondante pour nourrir les habitants de ce pays ${ }^{35}$.» Alors, même si la colonie présentait un climat hivernal plus froid que celui de la mère patrie, cela ne nuisait pas nécessairement à la mise en culture des terres. En effet, malgré les contraintes évidentes qui pesaient sur la production paysanne, les habitants de la colonie réussissaient à récolter suffisamment de blé pour subvenir aux besoins locaux la plupart du temps. Alors, le climat colonial différait de celui de la mère patrie, mais n'empêchait nullement son développement.

\section{EXPLIQUER LE CLIMAT}

Plusieurs observateurs ne se contentaient pas de décrier les différences mais s'efforçaient aussi d'en trouver les causes. La latitude n'expliquant guère les différences, les Français proposèrent donc diverses explications, comme une topographie distincte de celle de la colonie. Selon certains, les montagnes du nord, les rivières plus nombreuses et, surtout, les forêts plus étendues causaient les différences.

Biard posa la question: «quelle peut estre la cause de ces frimas, $\&$ de ce plus grand froid, que nous ne sentons d'ordinaire en France?» Il rejeta l'influence déterminante de la latitude sur le climat dans la pensée contemporaine: «les causes n'en estans au ciel, il les faut rechercher en terre». Après avoir considéré plusieurs possibilités, il proposa deux raisons majeures: «nous n'avons trouvé que deux causes de la disproportion qu'il y a entre ce pays là, $\&$ cestui-cy, quant au temps $\&$ saisons : l'une est, que Canada est plus Aquatique : \& l'autre quell'est inculte». Comme celle de plusieurs observateurs, sa description du climat dépendait d'une certaine connaissance cartographique de la Nouvelle-France. Biard considéra les vents, mais il mit finalement l'accent sur la présence de l'eau et le fait que

33. R. G. Thwaites, dir., Jesuit Relations..., op. cit., vol. 36, 164.

34. Ibid., vol. 50, 244.

35. Voyages et mémoires sur le Canada par Franquet (Québec: Imprimerie générale A. Coté et Cie, 1889), $179-180$. 
la terre n'avait pas encore été cultivée ${ }^{36}$. Au XVII ${ }^{e}$ siècle, les Jésuites rapportèrent plusieurs fois la même explication: «L'experience nous fait voir que les bois engendrent les frimas \& les gelées ${ }^{37}$. " Au XVIII ${ }^{e}$ siècle, Charlevoix passa en revue les raisonnements des autres auteurs, pour finalement en arriver à identifier l'impact du Grand Nord: «il faut s'en tenir au voisinage des Glaces du Nord ${ }^{38}$ ».

Pour l'auteur anonyme de L'Histoire chronologique de la Nouvelle France, la colonie n'offrait pas de grande promesse en termes de richesse à part les fourrures. Même les ressources de la forêt étaient limitées: «le bois n'y est pas de consequence vû qu'il n'est pas assez cuit par le soleil ce qui fait qu'il n'est pas fort propre à batir des navires ${ }^{39}$ ". Nicolas-Gaspard Boucault amena deux raisons pour expliquer le climat local: primo, la présence de la forêt, ce qui voulait dire que «les neiges s'y conservent plus long tems et augmentent le froid en communiquant leur fraîcheur glaciale aux vents qui passent par dessus». Secundo, l'impact du vent du nordouest, "qui est d'un froid insupportable et qui règne continuellement pendant cette saison [1'hiver] $»^{40}$. Certes, les commentateurs savaient que l'on ne pouvait guère influencer les vents. Mais une des explications pour le climat différent de la Nouvelle-France portait en elle-même une proposition pour le changer.

\section{CHANGER LE CLIMAT}

Si le climat répondait surtout à la présence de la forêt ancienne dans la vallée du Saint-Laurent, la mise en culture de la terre devait ipso facto le modifier. En hiver, les arbres abritaient la neige, et elle y restait plus longtemps que dans les champs découverts. La coupe des arbres devait exposer la neige à la chaleur du soleil et la faire fondre plus rapidement. Du point de vue des micro-climats, il est certain que le défrichement sert à rechauffer la terre. Tout cultivateur pouvait bien constater ce genre de changement. Pour les contemporains, l'effet cumulatif des changements d'ordre micro devait égaler à un changement macro du système. Ils géné-

36. R. G. Thwaites, dir., Jesuit Relations..., op. cit., vol. 3, 56, 46, 58.

37. Ibid., vol. 5, 182 .

38. P. de Charlevoix, Journal d'un voyage..., op. cit., t. 3, 170.

39. Histoire chronologique de la Nouvelle France ou Canada depuis sa découverte (Mil Cinq Cents Quatre) juques en l'an Mil Six Cents Trente Deux (Montréal, Éditions Élysées, 1975), 3. La maison d'édition attribue ce livre à la plume de Xiste Le Tac, mais comme l'indique la notice du Dictionnaire biographique du Canada, cette attribution ne se tient pas. Michel Paquin, «Le Tac, Xiste», Dictionnaire biographique du Canada en ligne, ww.biographi.ca/FR [consulté le 14 mai 2015].

40. "État présent du Canada...", loc. cit., 23. 
ralisaient une influence observable à une échelle locale à une échelle géographique beaucoup plus vaste. Leur approche s'apparentait à celle de leurs collègues d'autres pays européens: les Espagnols et les Anglais, par exemple, croyaient eux aussi que le déboisement devait modifier les climats coloniaux ${ }^{41}$.

En Nouvelle-Angleterre, les colons ont bien remarqué vers la fin du XVIII ${ }^{e}$ siècle l'effet local des défrichements. Une fois les arbres abattus, les champs devenaient plus chauds et secs. Samuel Williams dans son History of Vermont, publié en 1809, affirma: «the earth and the air, in the cultivated parts of the country, are heated in consequence of their cultivation, ten or eleven degrees [Fahrenheit] more, than they were in their uncultivated state $^{42}$."Selon l'historien Jan Golinski, les penseurs de l'époque de Lumières des deux côtés de l'Atlantique célébraient l'impact des actions humaines qui, selon eux, avaient pour effet de "civiliser» la nature, soit de modérer les extrémités du climat nord-américain ${ }^{43}$.

Mais en Nouvelle-France, cette approche apparut dès le XVII ${ }^{\mathrm{e}}$ siècle. Le jésuite Biard fit ce lien entre l'impact du défrichement local et un changement climatique plus important:

Là où si la terre estoit habitée, $\&$ cultivée, outre que d'elle, $\&$ des logis des habitans monteroyent des exhalations, c'est à dire, des fumées chaudes, \& seches: le soleil de plus la trouveroit disposée à sentir ses rayons, $\&$ dissipper le froit, $\&$ broüillars: ce qui nous estoit fort oculaire, $\&$ sensible. Car en ce peu que nous avions labouré, tousiours la nege s'y fondoit plustost qu'autre part, $\&$ de là d'ordinaire les broüees commençoyent à se dissiper, $\&$ peu à peu s'esvanouir $^{44}$.

En 1633, le père Le Jeune se montra d'accord avec cette explication: « $\mathrm{Si}$ le pays estoit découvert jusques à ces montagnes; nous aurions peutestre l'une des plus foecondes vallées qui soient en l'univers ${ }^{45}$."

En 1665, le jésuite François Le Mercier se souvint de la promesse du roi français de «changer nos forests en villes, \& nos deserts en Provinces...

41. Brant Vogel, "The Letter from Dublin: Climate Change, Colonialism, and the Royal Society in the Seventeenth Century", Osiris, 26 (2011): 114.

42. Williams, cite dans William Cronon, Changes in the Land: Indians, Colonists, and the Ecology of New England, édition révisée (New York, Hill and Wang, 2003), 122.

43. J. Golinski, «American Climate», loc. cit., 169-70. Pour le cas en France, consulter René Favier, "Penser le changement climatique au siècle des Lumières", dans Denis Lamarre, Climat et risques. Changement d'approches (Paris, Lavoisier, 2008), 9-23, \{halshs-00375761\}, https:/ / halshs.archives-ouvertes. $\mathrm{fr} /$ halshs-00375761 [consulté le 14 mai 2015].

44. R. G. Thwaites, dir., Jesuit Relations..., op. cit., vol. 3, 60.

45. Ibid., vol. 5, 182 . 
$[\mathrm{P}]$ uisque ces terres sont au mesme climat que la France, elles auront aussi la mesme benignité de l'air, quand on pourra les cultiver $\&$ les descharger de leurs bois. " L'avenir était plein de promesses: la Nouvelle-France deviendrait « un païs, qui ne cedera en rien, pour la fertilité de la terre, $\&$ pour la douceur du climat, à ce qui se trouve de plus doux, $\&$ plus aimable en Europe $»^{46}$. Charlevoix constata que l'influence du Grand Nord allait perdurer. Néanmoins, «si le Canada étoit aussi découvert $\&$ aussi peuplé que la France, les Hyvers y seroient moins long $\&$ moins rudes ${ }^{47}$."

$\mathrm{Au} \mathrm{XVIII}{ }^{e}$ siècle, les observateurs conclurent que la vallée du SaintLaurent devenait de plus en plus chaude et ils attribuaient ce changement à l'effet du défrichement cumulatif de la région. Le défrichement transformait le paysage laurentien de façon remarquable. Alain Laberge a calculé, à l'aide des aveux et dénombrements, qu'environ 150000 arpents se trouvaient soit en labour ou en prairies dans la vallée du Saint-Laurent vers 1725 , c'est-à-dire seulement deux générations après le grand flot migratoire de la fin du XVII ${ }^{e}$ siècle. Dans les gouvernements de Montréal et de Québec, presque la moitié des terres sous contrôle seigneurial était déjà exploitée ${ }^{48}$.

Pour les contemporains, ce défrichement eut un impact clair. Le botaniste finnois Pehr Kalm relata la perspective populaire. Un homme de plus de 80 ans qu'il avait rencontré près de Trois-Rivières proposa, comme plusieurs, que les hivers étaient plus sévères dans sa jeunesse: «les hivers étaient infiniment plus froids que ceux de maintenant... Il tombait autrefois beaucoup plus de neige que maintenant...; il affirme en outre que les étés de maintenant sont beaucoup plus chauds que ceux de sa jeunesse ${ }^{49}$." Comme le remarque l'historien Brant Vogel, avec le vieillissement, la mémoire humaine tend à exagérer la dûreté climatique vécue au cours de la jeunesse $\mathrm{s}^{50}$. Néanmoins, pour plusieurs contemporains, les tempéra-

46. Ibid., vol. 49, 212 ; vol. 50, 88 .

47. P. de Charlevoix, Journal d'un voyage..., op. cit., 170.

48. Alain Laberge, dir., Portraits de campagnes: La formation du monde rural laurentien au XVIII siècle (Québec, Les Presses de l'Université Laval, 2010), 57-58.

49. Jacques Rousseau, Guy Béthune et Pierre Morisset, trad. et dir., Voyage de Pehr Kalm au Canada en 1749 (Montréal, Pierre Tisseyre, 1977), 421. Selon Fredrik Albritton Jonsson, Pehr Kalm a rapporté des témoignages très différents concernant le changement du climat dans les colonies britanniques nordaméricaines. Il s'est montré beaucoup plus positif envers la Nouvelle-France, qu'il a d'ailleurs considérée beaucoup plus érudite. "Climate Change and the Retreat of the Atlantic: The Cameralist Context of Pehr Kalm's Voyage to North America, 1748-51 ", William and Mary Quarterly, $3^{\mathrm{e}}$ série, 72, 1 (janvier 2015): 99-126.

50. B. Vogel, «The Letter from Dublin...», loc. cit., 114. 
tures se sont réchauffées au XVIII ${ }^{\mathrm{e}}$ siècle par rapport au XVII ${ }^{\mathrm{e}}$ siècle, ce qui va dans le même sens des reconstructions climatiques.

Vers les années 1740, le médecin Jean-François Gaultier introduisit dans la colonie des méthodes scientifiques pour mieux mesurer les températures quotidiennes. Utilisant un thermomètre, Gaultier enregistra pendant plusieurs années les températures du matin à Québec, en notant de façon subjective si le temps était "beau», "couvert», "doux», etc. Il envoya ses notes à Henri Louis Duhamel du Monceau dont certaines furent publiées dans les Mémoires de l'Académie royale des sciences. Ce dernier faisait aussi état d'une connaissance populaire d'un réchauffement: «Les personnes les plus âgées ne se souviennent point d'avoir vû un hiver si doux, aussi le fleuve Saint-Laurent n'a-t-il pas pris cette année vis-à-vis de Quebec ${ }^{51}$.» D’ailleurs, selon Victoria Slonosky, les données de Gaultier nous permettent d'affirmer que les températures se sont bien réchauffées vers le milieu $\mathrm{du} \mathrm{XVIII}^{\mathrm{e}}$ siècle, pour devenir similaires à celles du milieu du $\mathrm{XX}^{\mathrm{e}}$ siècle ${ }^{52}$.

Ce réchauffement fournissait des preuves convaincantes des bienfaits de la colonisation de la Nouvelle-France. Gaultier affirmait que certains associaient le défrichement au changement climatique: «Les habitans du Canada prétendent que les hivers ne sont plus si rigoureux qu'ils étoient anciennement, ce qu'ils attribuent à la grande quantité de terre qu'on a défrichée ${ }^{53}$.» Kalm, qui rencontra Gaultier lors de son voyage en NouvelleFrance, fut d'accord. Il rapporta que certains contemporains attribuèrent le changement à l'effet du défrichement:

Différents prêtres me disent que depuis l'époque où on a commencé de mettre en culture le Canada, les étés sont devenus plus longs, qu’ils surviennent plus tôt et se prolongent davantage; par contre, les hivers durent moins longtemps que dans l'ancien temps ${ }^{54}$.

Pour le philosophe le comte de Volney, qui a écrit un ouvrage détaillé sur le climat nord-américain au XVIII ${ }^{e}$ siècle, l'impact de l'action humaine sur le climat tant au Canada qu'aux États-Unis était inéluctable:

51. H. L. duHamel du Monceau, «Observations botanicos-météorologiques faites en Canada, par M. Gautier...", Mémoires de l'Académie Royale des Sciences (1747): 470.

52. Victoria C. Slonosky, «The Meteorological Observations of Jean-François Gaultier, Quebec, Canada: 1742-56", Journal of Climate, 16 (juillet 2003): 2237.

53. H. L. duHamel du Monceau, "Observations botanicos-météorologiques faites à Québec pendant les mois d'Octobre, Novembre \& Décembre 1744, \& les mois de Janvier, Février, Mars, Avril \& Mai 1745 », Mémoires de l'Académie Royale des Sciences (1746): 91.

54. J. Rousseau et al., Voyage de Pehr Kalm..., op. cit., 469. 
depuis les premiers temps de la colonie du Canada, les époques de la clôture du fleuve par les glaces, ont retardé de près d'un mois, et qu'au lieu d'assurer les vaisseaux sous la condition d'être sortis à la fin de novembre, comme il était spécifié au commencement du siècle dernier, la clause actuelle d'assurance n'a plus lieu que pour le vingt-cinq décembre...

Pour lui, comme pour plusieurs des habitants de la colonie, ce changement représentait une "amélioration" qui devait accompagner la coupe des forêts, «qui ferment le passage au fleuve aërien $»^{55}$.

\section{CONCLUSION}

Dans la perspective de l'histoire du climat, les plus importants mouvements migratoires français vers la Nouvelle-France eurent lieu au moment du Minimum de Maunder. Entre 1660 et 1720, en Amérique du Nord aussi bien qu'en Europe, le climat était plus froid - en général d'un degré Celsius - en comparaison du $\mathrm{XX}^{\mathrm{e}}$ siècle. Même si nous acceptons que les deux continents connurent des périodes froides, la Nouvelle-France expérimenta des hivers encore plus froids et plus longs. Les Français s'efforcèrent d'expliquer les grandes différences entre la colonie et la mère patrie. Pour eux, le climat semblait peu refléter l'occupation préalable des sols par les Autochtones. Plutôt, ce furent les grandes forêts qui expliquaient le froid soutenu, ainsi que les vents du nord. En revanche, le défrichement progressif des terres dans la vallée du Saint-Laurent offrait la promesse de mitiger le froid hivernal et ainsi de prolonger l'été dont dépendait l'agriculture d'inspiration européenne, essentiellement basée sur le blé.

Vers les années 1740 , certains habitants de la colonie constataient en effet l'adoucissement du climat. Voilà la preuve que le défrichement devait améliorer le climat. Les défrichements, qui eurent sans doute un impact très local, servaient aussi à expliquer les impacts à une échelle beaucoup plus grande. Les habitants ont donc anticipé ce que les historiens de l'environnement appelleraient aujourd'hui l'impact anthropogène: l'action humaine devait changer l'environnement de façon radicale. Mais ils avaient tort. Le climat du XVIII ${ }^{\mathrm{e}}$ siècle s'adoucissait à cause des effets naturels, tels l'activité solaire ou volcanique, et le réchauffement des températures moyennes s'étendait bien au-delà de la colonie, pour avoir un impact aussi dans la mère patrie. Alors, en croyant que le simple défrichement des terres agricoles allait améliorer les conditions, les habitants de

55. C.-F. Volney, Tableau du climat et du sol des Etats-Unis d'Amérique, suivi d'éclaircissemens sur la Floride, sur la colonie Française au Scioto, sur quelques colonies Canadiennes et sur les Sauvages, (Paris, Chez Courcier et Dentu, 1803), 227-228. 
la Nouvelle-France ont surestimé leur impact sur le climat colonial. Néanmoins, vers le milieu du XVIII ${ }^{\mathrm{e}}$ siècle, plusieurs personnes étaient bel et bien convaincues que leurs actions étaient à la source de l'adoucissement du climat en Nouvelle-France. 